\title{
Action du phloroglucinate de pipérazine à l'égard de divers Helminthes parasites des Bovidés
}

\author{
por J. GUILHON ef M. GRABER
}

L'action anthelminthique du phloroglucinate de pipérazine qui s'exerce à l'égard de divers Cestodes et Nématodes parasites de plusieurs espèces animales domestiques (poulef, mouton, âne) fut aussi étudiée sur les helminthes observés dans le tractus digestif des Bovidés en Afrique tropicale (Tchad).

\section{MATÉRIEL. ET MÉTHODE}

Le protocole expérimental de base fut identique à celui que nous avons employé dans les recherches effectuées sur les ânes de 1959 à 1962. Toutefois, deux opérations furent ajoutées pour mieux suivre les variations du taux parasitaire des Trichostrongylidés de faibles dimensions (Cooperia). Des coprocultures préparées avant et après le traitement jusqu'au jour de l'autopsie permettent de mieux apprécier la présence ou l'absence des Cooperia et des larves du troisième âge de Bunostomum phlebotomum, Oesophagostomum radiatum et d'Haemoncus contorfus.

Enfin, immédiatement après la sacrification, des prélèvements obtenus par raclage de muqueuse d'intestin grêle examinés au microscope entre lame et lamelle permettent de contrôler, plus sûrement, la présence ou l'absence de Cooperia.
31 bouvillons, dont 10 témoins, parasités par 12 espèces d'helminthes furent utilisés pour l'expérimentation. Dans l'organisme de 90 p. 100 d'entre eux, les vers ci-dessous indiqués se trouvaient associés par deux ou trois espèces, plus rarement par 5 ou 6 :

Schistosoma bovis
Poramphistomum microbothrium
Moniezia benedeni
Thysaniezia ovilla
Cysticercus bovis
Oesophagostomum radiatum
Bunostomum phlebotomum
Hoemoncus contortus
Cooperia punctata
Cooperia pectinato
Cooperia sp.
Setaria labioto-popillosa

Le phloroglucinate de pipérazine en suspension dans l'eau a été administré, à la bouteille, aux bovins à jeun depuis 20 d̀ 24 heures, aux doses de 200 à $600 \mathrm{mg} / \mathrm{kg}$, en une seule fois, ou de 250 à $300 \mathrm{mg} / \mathrm{kg}$ répétées deux fois à 24 heures d'intervalle. Les résultats obtenus sont groupés dans les dix tableaux ci-inclus, complétés par des commentaires.

Io Action sur les Trématodes.

TABLEAU no I. - Pourcentage de réduction du nombre de trématodes.

\begin{tabular}{|c|c|c|c|c|c|}
\hline \multirow{2}{*}{ Helminthes en cause } & \multicolumn{3}{|c|}{ Doses uniques $(\mathrm{mg} / \mathrm{kg})$} & \multirow{2}{*}{$\begin{array}{l}\text { Doses répélées } 2 \text { fois } \\
\text { à } 24 \text { heures d'inter- } \\
\text { valle }(\mathrm{mg} / \mathrm{kg}) \\
\frac{300}{}\end{array}$} & \multirow{2}{*}{$\begin{array}{c}\text { Témoins } \\
\text { Poids de } \\
\text { parasites } \\
\text { (gr) }\end{array}$} \\
\hline & 200 & 400 & 600 & & \\
\hline $\begin{array}{l}\text { Schistosama bovis } \\
\text { Paromphistomum microbothrium }\end{array}$ & 0 p. $100^{*}$ & 0 p. $100 * *$ & 0 p. $100 * *$ & 0 p. $100 * * * * *$ & $1 \mathrm{~g}$ \\
\hline
\end{tabular}

Rev. Elev. Méd. vét. Pays trop. 1963, 16, no 3

Reçu pour publication : juillet 1963. 
Aucune diminution du nombre d'œufs au phistomes étaient présents dans la panse des gr n'a 'été enregistréé daniș le 'ca's où les Param-. sujets traités.

\section{$2^{\circ}$ Action súr les Cestodes.}

TABLEAU no II. - Pourcentagé de réduction du nombire de parasites après autopsie.

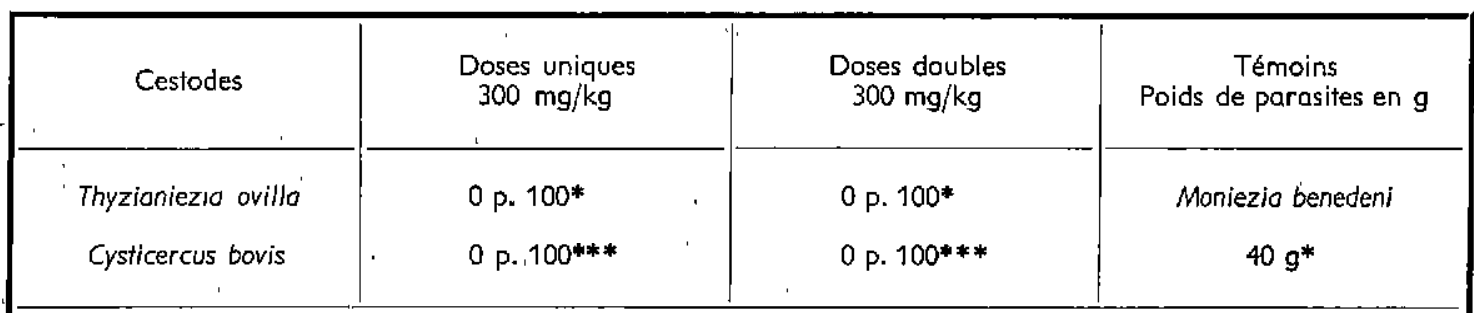

* = Un animal parasité.

\section{Action sur les Nématodes.}

A. - Action sur Oesophagostomum radiatum, Bunostomum phlebotomum et Haemoncus contortus.

TABLEAU no III. - Haemoncus contortus, Oesophagostomum rodiotum, Bunostomum phlebotum; nombre d'ceufs au g (moyenne)

\begin{tabular}{|c|c|c|c|c|}
\hline Doses ( $\mathrm{mg} / \mathrm{kg}$ ) & Avant traitement & Après traitement' & Restent & $\begin{array}{l}\text { Pourcentage } \\
\text { d'efficacilé }\end{array}$ \\
\hline \multicolumn{5}{|l|}{ Doses uniques : } \\
\hline 200 & 250 & 150 & Bunostomum phlebotomum & 40 p. 100 \\
\hline 300 & 315 & 0 & $\begin{array}{l}\text { Haemoneus contorfus } \\
\text { Haemancus contortus }\end{array}$ & 100 p. 100 \\
\hline $\begin{array}{l}400 \\
600\end{array}$ & $\begin{array}{l}622 \\
582\end{array}$ & $\begin{array}{l}84 \\
84\end{array}$ & $\begin{array}{l}\text { Bunostomum phlebofomum } \\
\text { Bunostomum phlebotomum }\end{array}$ & $\begin{array}{l}87 \text { p. } 100 \\
86 \text { p. } 100\end{array}$ \\
\hline \multicolumn{5}{|c|}{ Doses répetees deux fois ò 24 heures d'intervalle: } \\
\hline $\begin{array}{l}250 \\
300\end{array}$ & $\begin{array}{l}105 \\
472\end{array}$ & $\begin{array}{r}0 \\
105\end{array}$ & $\begin{array}{l}\text { Bunostomum phlebolomum } \\
\text { Bunostomum phiebotomum } \\
\text { Haemoncus contortus }\end{array}$ & $\begin{array}{r}100 \text { p. } 100 \\
78 \text { p. } 100\end{array}$ \\
\hline
\end{tabular}

TABLEAU no IV. - Pourcentage de réduction du nombre de Nématodes après autopsie.

\begin{tabular}{|c|c|c|c|c|c|c|c|}
\hline \multirow{2}{*}{$\begin{array}{l}\text { Porasites en } \\
\text { cause }\end{array}$} & \multicolumn{4}{|c|}{ Doses uniques $(\mathrm{mg} / \mathrm{kg})$} & \multicolumn{2}{|c|}{ Doses doubles $(\mathrm{mg} / \mathrm{kg})$} & " \\
\hline & 200 & 300 & 400 & 600 & 250 & 300 &  \\
\hline \multirow{3}{*}{$\begin{array}{l}\text { Oesophagosto- } \\
\text { mum radiatum } \\
\text { Haemoncus } \\
\text { contortus } \\
\text { Bunostomum } \\
\text { phlebotomum }\end{array}$} & 100 p. $100^{*}$ & 100 p. $100^{* * *}$ & 100 p. $100^{* * *}$ & 100 p. $100 * * *$ & $=100$ p. $100^{* * *}$ & 100 p. $100 * * * * * * *$ & $\left\{\begin{array}{c}47 * * * * * \\
* *\end{array}\right.$ \\
\hline & 0 p. 100* & 94 p. $100 * * * *$ & 100 p. $100 * *$ & 100 p. $100 *$ & 100 p. $100 * *$ & 67 p. $100^{* * * * * * *}$ & $\mid 120 * * * *$ \\
\hline & Op. 100* & 25 p. $100 * *$ & 6 p. $100 *$ & 10 p. $100 * * *$ & 2 p. $100^{*}$ & 0 p. $100^{* * * *}$ & $\underset{* *}{13^{* * * *}}$ \\
\hline
\end{tabular}


B. - Action sur les Cooperio.

TABLEAU no $\mathrm{V}$. - Action du phloroglucinate de pipérazine sur Cooperia pectinato et Cooperia punctato

\begin{tabular}{|c|c|c|c|c|c|c|c|c|}
\hline \multirow[t]{2}{*}{$\begin{array}{l}\text { Doses } \\
(\mathrm{mg} / \mathrm{kg})\end{array}$} & \multicolumn{2}{|c|}{$\begin{array}{c}\text { Examens } \\
\text { coprologiques. } \\
\text { Nombre d'œufs } \\
\text { au gr }\end{array}$} & \multicolumn{2}{|c|}{$\begin{array}{l}\text { Culture d'œufs. } \\
\text { Présence de larves }\end{array}$} & \multirow[t]{2}{*}{$\begin{array}{l}\text { Grattage de } \\
\text { muqueuses }\end{array}$} & \multirow{2}{*}{$\begin{array}{c}\text { Nombre } \\
\text { de } \\
\text { parasites } \\
\text { à } \\
\text { l'cu- } \\
\text { topsie }\end{array}$} & \multirow{2}{*}{$\begin{array}{c}\text { Nombre } \\
\text { d'ani- } \\
\text { maux } \\
\text { toiale- } \\
\text { ment } \\
\text { dépara- } \\
\text { sités }\end{array}$} & \multirow{2}{*}{$\begin{array}{l}\text { Témoins } \\
\text { Nombre de } \\
\text { parasites. } \\
\text { Moyenne }\end{array}$} \\
\hline & Av. trait. & Ap. trait. & Av. trait. & Ap. trait. & & & & \\
\hline \multicolumn{9}{|c|}{ Doses uniques } \\
\hline $\begin{array}{l}200^{*} \\
300^{* *} \\
400 * * \\
600^{* * *}\end{array}$ & $\begin{array}{r}84 \\
105 \\
\\
320 \\
168\end{array}$ & $\begin{array}{r}84 \\
0 \\
84 \\
0\end{array}$ & 1 sur 2 & $\begin{array}{l}\text { Absence } \\
2 \text { sur } 2\end{array}$ & $\begin{array}{l}\text { Formes jeunes } \\
\text { immatures }\end{array}$ & $\begin{array}{r}20 \\
5 \\
30 \\
0\end{array}$ & $\left\{\begin{array}{c}0 \\
0 \\
1 \text { sur } 2 \\
2 \operatorname{sur} 2\end{array}\right.$ & $\begin{array}{l}\text { Cooperia Pecti- } \\
\text { nata }+ \text { Cooperia- } \\
\text { punctata }=40\end{array}$ \\
\hline \multicolumn{9}{|c|}{ Doses répétées deux fois ò 24 heures d'intervalle } \\
\hline $\begin{array}{l}250^{* * *} \\
300^{* * *}\end{array}$ & $\begin{array}{c}0 \\
682\end{array}$ & $\begin{array}{r}0 \\
55\end{array}$ & $\begin{array}{l}\text { Présence } \\
2 \text { sur } 2 \\
\text { Présence } \\
3 \text { sur } 3\end{array}$ & $\begin{array}{l}\text { Absence } \\
1 \text { sur } 2 \\
\text { Présence } \\
3 \text { sur } 3\end{array}$ & $\begin{array}{l}\text { Formes adultes } \\
\text { Formes adultes }\end{array}$ & $\begin{array}{l}10 \\
12\end{array}$ & $\begin{array}{c}1 \text { sur } 2 \\
0\end{array}$ & ' \\
\hline
\end{tabular}

* $=$ Un animal parasité.

TABLEAU no VI. - Pourcentage de réduction du nombre d'CEsophagostomes après traitement avec quatre dérivés de la pipérazine

\begin{tabular}{|c|c|c|c|c|}
\hline $\begin{array}{l}\text { Doses } \\
(\mathrm{mg} / \\
\mathrm{kg})\end{array}$ & $\begin{array}{c}\text { Dilauraìe } \\
\text { de } \\
\text { pipérazine }\end{array}$ & $\begin{array}{c}\text { Citrate } \\
\text { de } \\
\text { pipérazine }\end{array}$ & $\begin{array}{l}\text { Dithiocar- } \\
\text { bamate de } \\
\text { pipérazine }\end{array}$ & $\begin{array}{l}\text { Phloroglu- } \\
\text { cinate de } \\
\text { pipérazine }\end{array}$ \\
\hline \multicolumn{5}{|c|}{ Doses uniques : } \\
\hline $\begin{array}{l}100 \\
150 \\
180 \\
200 \\
250 \\
300 \\
400 \\
600\end{array}$ & $\begin{array}{l}100 \text { p. } 100 \\
100 \text { p. } 100 \\
100 \text { p. } 100\end{array}$ & $\begin{array}{l}80,7 \text { p. } 100 \\
100 \text { p. } 100 \\
100 \text { p. } 100\end{array}$ & $\begin{array}{r}100 \text { p. } 100 \\
97,6 \text { p. } 100 \\
100 \text { p. } 100\end{array}$ & $\begin{array}{l}100 \text { p. } 100 \\
100 \text { p. } 100 \\
100 \text { p. } 100 \\
100 \text { p. } 100\end{array}$ \\
\hline \multicolumn{5}{|c|}{ Doses répétées deux fois à 24 heures d'intervalle: } \\
\hline $\begin{array}{l}250 \\
300\end{array}$ & & & ' & $\begin{array}{l}100 \text { p. } 100 \\
100 \text { p. } 100\end{array}$ \\
\hline
\end{tabular}

Dans tous les cas, que ce soit avec le Phloroglucinate, le dilaurate, le dithiocarbamate ou le citrate de pipérazine, une dose unique et relativement faible, de l'ordre de 150 d̀ $200 \mathrm{mg} / \mathrm{kg}$
TABLEAU ñ VII. - Pourcentage de réduction du nombre, de Bunostomes après traitement avec 4 dérivés pipéraziques

\begin{tabular}{|c|c|c|c|c|}
\hline $\begin{array}{c}\text { Doses } \\
(\mathrm{mg} / \\
\mathrm{kg})\end{array}$ & $\mid \begin{array}{c}\text { Dilaurate } \\
\text { de Pipéra- } \\
\text { zine }\end{array}$ & $\begin{array}{l}\text { Citrate de } \\
\text { Pipérazine }\end{array}$ & $\begin{array}{l}\text { Dithiocor- } \\
\text { bamate de } \\
\text { Pipérazine }\end{array}$ & $\begin{array}{l}\text { Phloroglu- } \\
\text { cinate de } \\
\text { Pipérazıne }\end{array}$ \\
\hline \multicolumn{5}{|c|}{ Doses uniques : } \\
\hline $\begin{array}{l}100 \\
150 \\
180 \\
200 \\
250 \\
300 \\
400 \\
600\end{array}$ & $\begin{array}{r}0 \text { p. } 100 \\
5.4 \text { p. } 100 \\
\text { o p. } 100\end{array}$ & $\begin{array}{l}\text { Op. } 100 \\
\text { op. } 100 \\
0 \text { p. } 100 \\
0 \text { p. } 100\end{array}$ & $\begin{array}{l}0 \text { p. } 100 \\
0 \text { p. } 100 \\
0 \text { p. } 100\end{array}$ & $\begin{array}{r}0 \text { p. } 100 \\
25 \text { p. } 100 \\
6 \text { p. } 100 \\
10 \text { p. } 100\end{array}$ \\
\hline \multicolumn{5}{|c|}{ Doses répéfées deux fois d 24 heures d'intervolle :' } \\
\hline $\begin{array}{l}250 \\
300\end{array}$ & &  & & $\begin{array}{l}2 \text { p. } 100 \\
0 \text { p. } 100\end{array}$ \\
\hline
\end{tabular}

est suffisante pour chasser les cesophagostomes.

Sur Bunostomum phlebotomum de 'I'intestin grêle des Bovidés, les quatre dérivés n'ont qu'une faible action même à doses élevées. 
TABLEAU $n^{0}$ VIII. - Pourcentage de réduction du nombre d'Haemoncus contortus après traitement avec 4 dérivés de la Pipérazine.

\begin{tabular}{|c|c|c|c|c|}
\hline $\begin{array}{l}\text { Doses } \\
(\mathrm{mg} / \\
\mathrm{kg})\end{array}$ & $\begin{array}{l}\text { Dithiocar- } \\
\text { bamate de } \\
\text { Pipérazine }\end{array}$ & $\begin{array}{l}\text { Citrate de } \\
\text { Pipérazine }\end{array}$ & $\left|\begin{array}{c}\text { Dilaurate } \\
\text { de Pipera- } \\
\text { zine }\end{array}\right|$ & $\begin{array}{l}\text { Phloroglu- } \\
\text { cinate de } \\
\text { Pipérazine }\end{array}$ \\
\hline \multicolumn{5}{|c|}{ Doses uniques : } \\
\hline $\begin{array}{l}100 \\
150 \\
180 \\
200 \\
250 \\
300 \\
400 \\
600\end{array}$ & $\left|\begin{array}{cc}6,5 & \text { p. } 100 \\
43 & \text { p. } 100 \\
21,6 & \text { p. } 100\end{array}\right|$ & $\begin{array}{r}27,4 \text { p. } 100 \\
24,5 \text { p. } 100 \\
9,4 \text { p. } 100 \\
8,9 \text { p. } 100\end{array}$ & 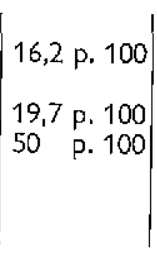 & $\begin{array}{r}0 \text { p. } 100 \\
94 \text { p. } 100 \\
100 \text { p. } 100 \\
100 \text { p. } 100\end{array}$ \\
\hline \multicolumn{5}{|c|}{ Doses répétées deux fois à 24 heures d'intervalle : } \\
\hline $\begin{array}{l}250 \\
300\end{array}$ & & & & $\begin{array}{r}100 \text { p. } 100 \\
67 \text { p. } 100\end{array}$ \\
\hline
\end{tabular}

Sur Hoemoncus contortus, trois dérivés : le dithiocarbamate, le Dilaurate et le Citrate de pipérazine ont une action assez irrégulière, alors que celle du Phloroglucinate est plus constante à partir de $300 \mathrm{mg} / \mathrm{kg}$. Les doses répétées deux fois à 24 heures d'intervalle ne donnent pas de meilleurs résultats que les doses uniques.

TABLEAU n०9. - Action des dérivés de la pipérazine sur Cooperia pectinata et $C$. punctata.

\begin{tabular}{|c|c|c|c|c|}
\hline $\begin{array}{c}\text { Doses } \\
(\mathrm{mg} / \mathrm{kg})\end{array}$ & $\begin{array}{c}\text { Dithio- } \\
\text { carba- } \\
\text { mate de } \\
\text { pipérazine }\end{array}$ & $\begin{array}{c}\text { Dilaurate } \\
\text { de pipera- } \\
\text { zıne }\end{array}$ & $\begin{array}{c}\text { Citrate de } \\
\text { pipéra- } \\
\text { zine }\end{array}$ & $\begin{array}{l}\text { Phloroglu- } \\
\text { cinate de } \\
\text { pipérazine }\end{array}$ \\
\hline \multicolumn{5}{|c|}{ Doses uniques : } \\
\hline $\begin{array}{l}100 \\
150 \\
180 \\
200 \\
300 \\
400 \\
600\end{array}$ & $\begin{array}{c}1 \text { sur } 2 \\
0 \\
0\end{array}$ & $\begin{array}{l}0 \\
0\end{array}$ & $\begin{array}{l}0 \\
0 \\
1 \\
0 \\
0\end{array}$ & $\begin{array}{c}0 \\
0 \\
1 \text { sur } 2 \\
2 \text { sur } 2\end{array}$ \\
\hline \multicolumn{5}{|c|}{ Doses répétées deux fois d̀ 24 heures d'intervalle : } \\
\hline $\begin{array}{l}250 \\
300\end{array}$ & & & & $\begin{array}{c}1 \text { sur } 2 \\
0\end{array}$ \\
\hline
\end{tabular}

Les dérivés de la pipérazine sont pratiquement sans action sur les diverses espèces de Cooperia. Toutefois, le Phloroglucinate à des doses élevées révèle une certaine activité dont la régularité n'a pu être suffisamment appréciée.

\section{MODE D'ÉLIMINATION}

Un grand nombre de Nématodes sont éliminés apparemment intacts dans les fèces. Il en est ainsi d'Oesophagostomum radiatum, Bunostomum phlebotomum ef Haemoncus contortus.

\begin{tabular}{|c|c|c|c|}
\hline & Hoemoncus & $\left|\begin{array}{c}\text { Esophogos- } \\
\text { tomum }\end{array}\right|$ & $\begin{array}{l}\text { Bunosio- } \\
\text { mum }\end{array}$ \\
\hline $\begin{array}{c}24 \text { heures après trai- } \\
\text { tement ........... } \\
48 \text { heures après trai- } \\
\text { tement ........... } \\
72 \text { heures après trai- } \\
\text { tement ............ } \\
96 \text { heures après trai- } \\
\text { tement ........... }\end{array}$ & $\begin{array}{l}33 \text { p. } 100 \\
58 \text { p. } 100 \\
90 \text { p. } 100\end{array}$ & $\begin{array}{l}50 \text { p. } 100 \\
38 \text { p. } 100 \\
12 \text { p. } 100\end{array}$ & $\begin{array}{l}50 \text { p. } 100 \\
50 \text { p. } 100\end{array}$ \\
\hline
\end{tabular}

Les Cooperia font exception à la règle ef leur expulsion ne peut être vérifiée correctement qu'après raclage de la muqueuse de l'animal sacrifié.

L'élimination commence dans les 24 heures pour se terminer environ 3 à 5 jours après le traitement. Le Phloroglucinate apparaît donc plutôt nématodifuge que nématodicide.

\section{TOXICITÉ}

Durant toutes les expériences jusqu'aux doses les plus élevées de $600 \mathrm{mg} / \mathrm{kg}$, aucun accident n'a été observé.

\section{CONCLUSION}

Le phloroglucinate de pipérazine agit sur les helminthes du bœuf comme nématodifuge faiblement polyvalent.

Son action est plus particulièrement nette sur Oesophagostomum radiatum du gros intestin et sur Hoemoncus contortus de la callette aux doses uniques de 300 à $400 \mathrm{mg} / \mathrm{kg}$.

Les autres Nématodes observés : Bunostomum phiebotomum, Cooperia pectinata ef Cooperia punctata sont plus résistants (600 $\mathrm{mg} / \mathrm{kg}$ ).

Enfin, le pouvoir anthelminthique du phloroglucinate de pipérazine à l'égard d'Hoemoncus contortus et d'Oesophagostomum rodiatum s'est montré supérieur à celui des trois autres dérivés (dithiocarbamate, dilaurate et citrate de pipérazine).

Ecole nationale vétérinaire d'Alfort Laboratoire de Parasitologie Laboratoire de recherches vétérinaires de Forcha-Fort-Lamy Service de Parasitologie. 


\section{SUMMARY}

The helmintic action of piperazine phloroglucinate on bovine nematodes.

Piperazine phloroglucinate is only feebly polyvalent as a nematodifuge in bovine helminthaisis. If is particularly effective against Oesophagostomum radiatum of the large intestine and on Haemonchus contortus of the abomasum in single dosage rates of $300-400 \mathrm{mg} / \mathrm{kg}$, but resistance is greater in infections of Bunostomum phlebotomum, Cooperia pectinota, and Cooperia punctata even at $600 \mathrm{mg} / \mathrm{kg}$.

It was shown that the anthelmintic properties of piperazine phloroglucinate with regard to Haemoncus contortus and Oesophogostomum rodiafum was superior to that other derivatives of piperazine viz. dithiocarbamate, dilaurate and citrate.

\section{RESUMEN}

Accion del floroglucinato de piperacina frente a diversos helmintos parasitos de los bovidos

El floroglucinato de piperacina actúa sobre los helmintos de los bóvidos como vermifugo de escasa polivalencia.

Su acción es mâs neta sobre Esofagostomun radiatum del intestino grueso y sobre Hemoncus confortus del cuajar a las dosis únicas de 300 à $400 \mathrm{mg} / \mathrm{kg}$.

Los otros nematodes observados : Bunostomun flebotomun, Cooperio pectinata y Cooperia puctato son môs resistentes $(600 \mathrm{mg} / \mathrm{kg})$.

Finalmente, el poder anti helmintíco del floroglucinato de piperacina frente a Hemoncus contortus y Esofagostomun rodiatun se ha mostrado superior al de los tres otros derivados (dithiocarbamato, dilaurato y citrato de piperacina). 